JOURNAL OF

APPLIED

CRYSTALLOGRAPHY

ISSN 1600-5767

\section{Optically Induced Nanostructures. Biomedical and Technical Applications. Edited by Karsten König and Andreas Ostendorf. De Gruyter, 2015. Pp. XL+329. Price Euro 119.95, USD 168, GBP 89.99.}

\author{
Nathalie Destouches* \\ University of Lyon - University Jean Monnet, UMR 5516 CNRS Laboratory Hubert Curien, 18 Rue Professeur B. Lauras, \\ F-42000 Saint-Etienne, France. *Correspondence e-mail: nathalie.destouches@univ-st-etienne.fr
}

Keywords: book reviews.

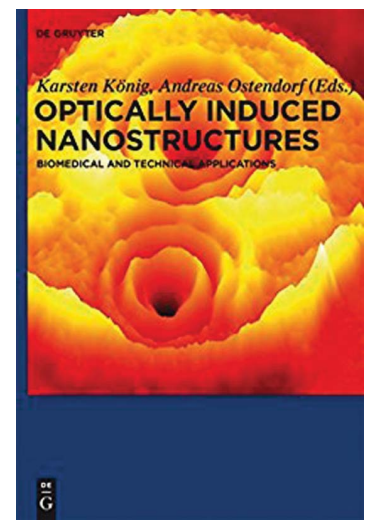

(C) 2017 International Union of Crystallography
Optically induced nanostructures. Biomedical and technical applications is an open-access book that any engineer, student or researcher interested in the use of ultrafast lasers for characterizing and engineering matter at the micro- and nanoscales should not miss. The book is available online at https://www.degruyter.com/view/product/212350. It gathers the recent achievements of 15 German research groups using mainly femtosecond nearinfrared lasers and classifies them into two parts: technical applications and biomedical applications. This book is not an exhaustive review, but it provides a relevant and broad interactions of ultrafast lasers with matter that shows how such laser-based techniques can reach a resolution smaller than the diffraction limit. The different chapters complement each other well, even though they are written as independent articles and their content may partly overlap. The overall content of the book is very cogent. Each chapter reports outstanding results that are representative of recent progress in each domain of application. They are, however, organized in different ways and do not pursue the same goals. Some of them give an overview of many examples of applications or nanostructures that can be reached with laser technology, others present recent characterization techniques, whereas most chapters focus on particular systems and describe with more detail their properties and/or the mechanisms behind their formation. The reader may be attracted by some specific subjects and can pick up here and there information on different materials, characterization techniques or processing conditions. The present review mainly aims to give the reader clues to find rapidly what he or she is looking for when opening this book.

Except for Chapter 7 in Part II, which could have appeared in Part I, the two parts of this book cover distinct topics. In Part I, one can learn about nanojets, two-photon threedimensional nanolithography, periodic self-organized surface and volume structures, nanodrilling, nanocutting, high-resolution characterization, and nonlinear imaging. Part II describes the use of ultrafast lasers for biomedical applications. This part shows how ultrafast lasers can be used to image cells with high spatial resolution, to modify cells at the nanoscale and to produce nanostructures on biocompatible substrates that can control the cell evolution. The last chapter is devoted to stimulated emission depletion (STED) techniques, which gained recognition after the Nobel Prize in Chemistry was received in 2014 by Stefan W. Hell (who developed STED microscopy in 2000), Eric Betzig and William E. Moerner for the development of super-resolved fluorescence microscopy. With this chapter the book concludes with results that perfectly highlight the high interest of all the technologies described therein, whose common aim is to reach sub$100 \mathrm{~nm}$ resolution with optical beams.

Although matter structuring with lasers is a story that is more than 50 years old, a lot of developments at the nanoscale have been achieved recently. Part I picks out the major applications in the field of materials science through the presentation of a series of particular studies. With Chapter 1, the reader is introduced to the high versatility of processing of high-repetition-rate sub-15 fs infrared lasers when tightly focused through a microscope. This chapter gives an interesting overview of micro- and nanostructuring techniques that can be implemented with ultrafast lasers. Chapter 2 focuses on the way to selection of information about imaging and structuring techniques based on nonlinear 
control the optical properties of nanojets, also called standing antennas, and of laser-induced periodic surface structures (LIPSS) generated on metal-coated silicon. LIPSS have been investigated on all kinds of materials since the 1960s, including metals, semiconductors and dielectrics. As in many chapters, we go through a broad topic via a very particular case, but with attention paid to the applications. Although quite specific, the studies of Chapter 2 include theoretical aspects that introduce general concepts used to model ultrafast laser-induced nanostructuring processes. Readers who are more interested in dielectric materials should look at Chapter 3, which specifically studies a particular case: the effect of temporal shaping of femtosecond pulses on generated sub-100 nm nanostructures. This chapter gives a good presentation of the fundamentals of light-matter interaction when considering dielectrics and pulse shaping. It also clearly describes spectral interferometry, a useful in situ characterization technique.

Chapter 4 deals with interferometric Fourier transform scatterometry, an optical characterization technique combined with numerical simulations that gives access to spatial frequencies below the diffraction limit. The link with the general topic of the book is in the fabrication process of plasmonic nanoantennae, which can considerably enhance the method resolution by transforming near-field information into far-field. A large part of this chapter is then dedicated to threedimensional two-photon polymerization, the technique used to produce the nanoantennae. The use of nanostructures to improve the spatial resolution of imaging systems is also the subject of Chapter 5. Here, the laser processing technique implemented to create useful nanostructures is not direct laser writing as in the previous chapter but laser-induced selforganization. Chapter 5 includes a parameter study for the generation of periodic nanostructures in bulk glass supported by both modelling and experiments. It also demonstrates how to use such buried nanostructures for high-resolution microscopy.

Chapter 6 reviews the progress in nonlinear processing of self-assembled monolayers with femtosecond lasers and introduces applications such as ultrathin resists and functional platforms for biochemical sensors. Chapter 7 is devoted to the application of LIPSS (already used in Chapter 2) to tribology. LIPSS are optimized here to reduce the friction coefficient of different kinds of materials.

Part II is then dedicated to biological and biomedical applications. Chapter 1 introduces techniques used to generate stem cells for regenerative therapies. It focuses on multiphoton microscopy with spectral and fluorescence lifetime imaging and also emphasizes the fact that ultrafast lasers are now used for cell nanosurgery and for cell reprogramming. With the next chapter, Chapter 1 is a good introduction for physicists to stem cells and their therapeutic issues.

In the following chapters, lasers are not directly used on cells anymore. Laser processing techniques similar to those already used in Part I are implemented to create biocompatible nanostructured materials. Chapter 2, after a large part on stem cells, describes the direct writing technique by multiplebeam interference and shows how laser-generated nanogrooves impact the development of cells. In Chapter 3, lasers are used to create metal nanoparticles by ablation. The latter are encapsulated in microgels immobilized on electrospun fibres to serve as controlled, programmed and adaptive multiple-ion-release wound dressings. Chapter 4 is a detailed description of a full study aimed at preparing microcarriers to influence the behaviour of cells. Here, LIPSS are produced by ultrafast laser treatment of a silicon wafer as an intermediate tool to produce alginate beads decorated with a controlled number of calcium phosphate nanoparticles, whose interaction with cells is studied. Chapter 5 proposes a thorough study of two-photon polymerization with an optimization of optical focusing and of the polymer itself. The versatile technique is applied to biomedical and microoptical applications, for which demanding geometries and specific optical properties are created. Chapter 6 also focuses on two-photon polymerization and pays special attention to the role of second harmonic generation by metallic nanoparticles in this process compared to two-photon absorption.

Chapter 7 presents an original structuring technology in which optically trapped particles are manipulated over the structured surface and used as masks in order to achieve arbitrary patterns. The potential and limitations of this method are described well through simulated and experimental results.

The last chapter is about STED microscopy and lithography, which are based on the use of two laser beams: one stimulates fluorescent molecules to glow, another cancels out all fluorescence except for that in a nanometre-sized volume. This allows one to bypass the Abbe resolution limit and gives optical microscopes access to the nanoworld. The chapter gives an introduction to STED microscopy and a state-of-theart review of STED lithography and deals with a particular example of application in biological and physiological research, the creation of nanoanchors for antibodies. 